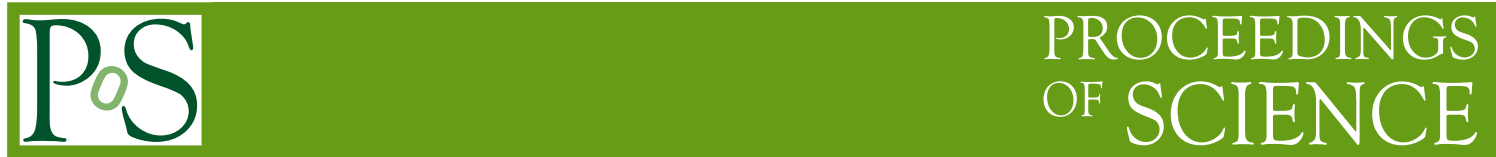

\title{
Supersymmetric Dark Matter in Light of Recent LHC Results
}

\author{
Keith A. Olive* \\ William I. Fine Theoretical Physics Institute, School of Physics and Astronomy, \\ University of Minnesota, Minneapolis, MN 55455, USA \\ E-mail: olive@umn.edu
}

\begin{abstract}
Recent LHC results, most notably on the Higgs mass, have had a major impact on the family of constrained supersymmetric models. The combination of the lack of discovery of supersymmetric particles at the LHC and a Higgs mass of $\sim 125 \mathrm{GeV}$, pushes the supersymmetric mass scales up considerably making the prospects of the direct detection of dark matter in cryogenic experiments more remote. After first drawing some relations between minimal supergravity models and the CMSSM, I will discuss some of the implications of the LHC results on the CMSSM and the prospects for direct detection.
\end{abstract}

VIII International Workshop on the Dark Side of the Universe,

June 10-15, 2012

Rio de Janeiro, Brazil

\footnotetext{
${ }^{*}$ Speaker.
} 


\section{Introduction}

One of the many appealing features attributed to the minimal supersymmetric extension of the standard model (MSSM) is the possibility for a dark matter candidate. If R-parity is preserved, the lightest supersymmetric particle (LSP) is stable and may make up the inferred dark matter in the Universe [1]. However, the MSSM, has over 100 undetermined parameters which makes a completely general analysis unfeasible. Therefore, it is common to make some well-motivated assumptions which relate many of these parameters, most of which are associated with supersymmetry breaking.

For example, in models based on minimal supergravity (that is models with a flat Kähler potential, mSUGRA), the low energy scalar potential can be written as $[2,3,4]$

$$
V=\left|\frac{\partial W}{\partial \phi^{i}}\right|^{2}+\left(A_{0} W^{(3)}+B_{0} W^{(2)}+\text { h.c. }\right)+m_{3 / 2}^{2} \phi^{i} \phi_{i}^{*}
$$

where $W$ is the superpotential for matter fields,

$$
W=\left(y_{e} H_{1} L e^{c}+y_{d} H_{1} Q d^{c}+y_{u} H_{2} Q u^{c}\right)+\mu H_{1} H_{2},
$$

when $\mathrm{SU}(2)$ indices have been suppressed. In (1.1), $W^{(3)}$ is the part of the superpotential cubic in fields, and $W^{(2)}$ is the part of the superpotential quadratic in fields, and $m_{3 / 2}$ is the gravitino mass. Thus, in mSUGRA models, one readily obtains scalar mass universality, $m_{0}=m_{3 / 2}$ (applicable at some input renormalization scale, $M_{\text {in }}$ usually associated with the grand unification scale). In addition, in mSUGRA, there is a relation between the tri- and bi-linear supersymmetry breaking terms, $B_{0}=A_{0}-m_{0}$. With a minimal choice for the gauge kinetic function, gaugino mass universality is also obtained. Furthermore, minimization of the Higgs potential leads to two conditions at the weak scale which can be expressed as

$$
\mu^{2}=\frac{m_{1}^{2}-m_{2}^{2} \tan ^{2} \beta+\frac{1}{2} m_{Z}^{2}\left(1-\tan ^{2} \beta\right)+\Delta_{\mu}^{(1)}}{\tan ^{2} \beta-1+\Delta_{\mu}^{(2)}},
$$

and

$$
B \mu=\left(m_{1}^{2}+m_{2}^{2}+2 \mu^{2}\right) \sin 2 \beta+\Delta_{B}
$$

where $m_{1,2}$ are the Higgs soft masses (evaluated at the weak scale), $\tan \beta$ is the ratio of the two Higgs vacuum expectation values, and $\Delta_{B}$ and $\Delta_{\mu}^{(1,2)}$ are loop corrections [5]. As a result, an mSUGRA model can be defined in terms of just 3 parameters: the gaugino mass, $m_{1 / 2}$ along with $m_{0}$ and $A_{0}$. In addition, the sign of the $\mu$ parameter must also be specified.

Often these strict mSUGRA relations are relaxed somewhat producing a slightly more general model known as the constrained MSSM (CMSSM) $[6,7,8,9,10]$. In the CMSSM, the relation between $A_{0}$ and $B_{0}$ is dropped allowing one to keep $\tan \beta$ as a free parameter, and while scalar mass universality is maintained, the relation between $m_{0}$ and the gravitino mass is usually dropped.

In what follows, I will briefly draw some relations between SUGRA models and the CMSSM and then concentrate on the impact of recent LHC results on the CMSSM parameter space. 

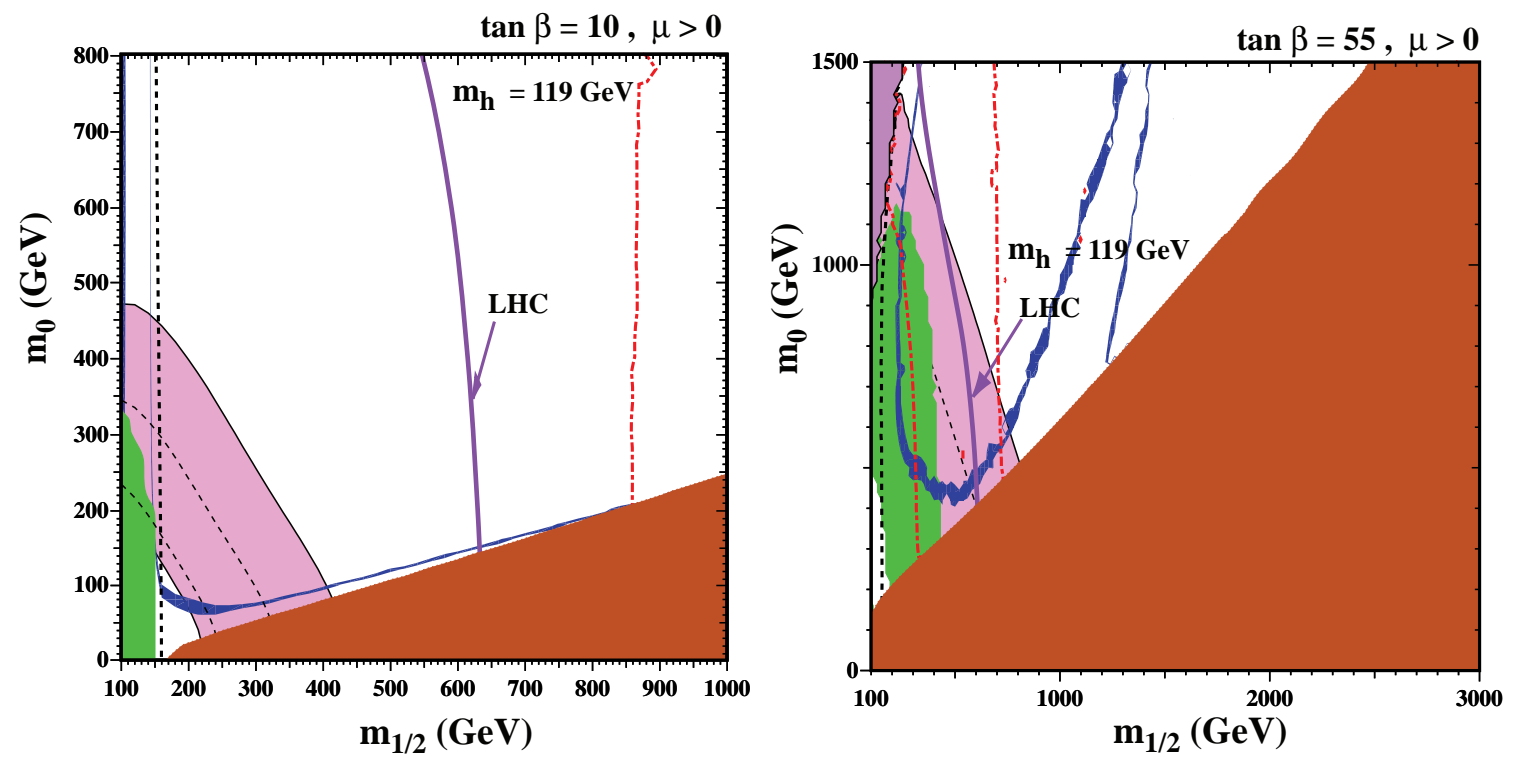

Figure 1: The $\left(m_{1 / 2}, m_{0}\right)$ planes for $(a) \tan \beta=10$ and $\mu>0$, assuming $A_{0}=0, m_{t}=173.1 \mathrm{GeV}$ and $m_{b}\left(m_{b}\right)_{S M}^{\overline{M S}}=4.25 \mathrm{GeV}$. The near-vertical (red) dot-dashed lines are the contours $m_{h}=119 \mathrm{GeV}$, and the near-vertical (black) dashed line is the contour $m_{\chi^{ \pm}}=104 \mathrm{GeV}$. The medium (dark green) shaded region is excluded by $b \rightarrow s \gamma$, and the dark (blue) shaded area is the cosmologically preferred region. In the dark (brick red) shaded region, the LSP is the charged $\tilde{\tau}_{1}$. The region allowed by the E821 measurement of $g_{\mu}-2$ at the 2- $\sigma$ level, is shaded (pink) and bounded by solid black lines, with dashed lines indicating the 1- $\sigma$ ranges. The curves marked LHC show the 95\% CL exclusion region (to the left of the curves) for LHC sparticle searches at 5/fb. In (b), $\tan \beta=55$. Here, in the upper left corner, the region with no EWSB is shaded dark pink.

\section{The CMSSM vs MSUGRA}

For given values of $\tan \beta, A_{0}$, and $\operatorname{sgn}(\mu)$, the regions of the CMSSM parameter space that yield an acceptable relic density and satisfy other phenomenological constraints may be displayed in the $\left(m_{1 / 2}, m_{0}\right)$ plane [10]. In Fig. 1a, the dark (blue) shaded region corresponds to that portion of the CMSSM plane with $\tan \beta=10, A_{0}=0$, and $\mu>0$ such that the computed relic density yields the WMAP value [11] of

$$
\Omega h^{2}=0.111 \pm 0.006
$$

Above the blue strip, annihilation cross sections are too small to maintain an acceptable relic density and $\Omega_{\chi} h^{2}$ is too large. The strip itself is produced by co-annihilation processes between the LSP and the next lightest sparticle (in this case the $\tilde{\tau}$ ) which enhance the annihilation cross section and reduce the relic density [12]. This occurs when the LSP and NLSP are nearly degenerate in mass. The dark (red) shaded region has $m_{\tilde{\tau}}<m_{\chi}$ and is excluded. Also shown in the figure are some phenomenological constraints from the lack of detection of charginos [13], as well as constraints from $b \rightarrow s \gamma$ [14] and $g_{\mu}-2$ [15]. Also shown and discussed in more detail below is the limit from LHC supersymmetry searches with $5 / \mathrm{fb}$ of data [16]. The locations of these constraints are described in the caption. 

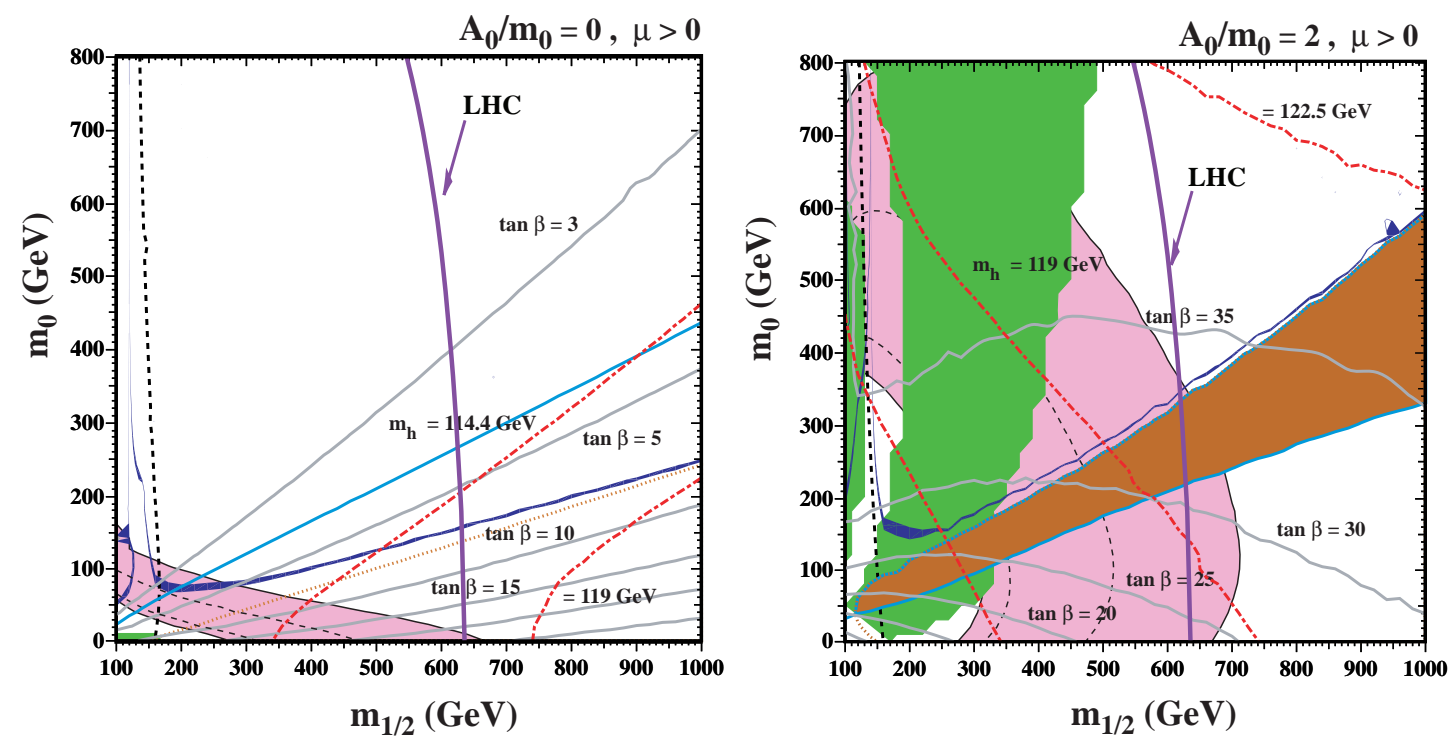

Figure 2: As in Fig. 1 for the minimal supergravity model with $A_{0} / m_{0}=0$ (left) and $A_{0} / m_{0}=2($ right $)$. The gravitino is the LSP below the diagonal light blue line and $m_{\tilde{\tau}}<m_{\chi}$ below the brown dotted curve. The contours for $\tan \beta$ are shown as solid gray curves.

At larger $m_{1 / 2}, m_{0}$ and $\tan \beta$, the relic neutralino density may be reduced by rapid annihilations through direct-channel $H, A$ Higgs bosons, as seen in Fig. 1(b) [6, 8]. Finally, the relic density can again be brought down into the WMAP range at large $m_{0}$ in the 'focus-point' region close the boundary where EWSB ceases to be possible and the lightest neutralino $\chi$ acquires a significant higgsino component [17]. The start of the focus point region is seen in the upper left of Fig. 1b.

In mSUGRA, the $\left(m_{1 / 2}, m_{0}\right)$ planes look very different as seen in Fig. $2[18,19]$. To begin with, once $A_{0} / m_{0}$ is specified, $\tan \beta$ must be computed at for each point in the plane. These are shown by the solid gray curves in increments of 5 as labeled. Also shown is the curve (solid, diagonal, light blue) for which $m_{3 / 2}=\min \left(m_{\chi}, m_{\tilde{\tau}}\right)$. Below this curve, the gravitino is the LSP. Another diagonal line (brown dotted) shows the contour for which the lightest neutralino mass $m_{\chi}$ is equal to the mass of the lighter stau, $m_{\tilde{\tau}_{1}}$. For $A_{0} / m_{0}=0$, the latter appears below the gravitino LSP line, and as such, $\tilde{\tau}_{1}$ is never the LSP. As a consequence, only the dark blue shaded region at low $m_{1 / 2}$ above the light blue line corresponds to neutralino dark matter at the WMAP density. The dark blue shaded region below the light blue line corresponds to the gravitino LSP at the WMAP density assuming that there is no nonthermal contribution to the gravitino density. Here, the gravitino density is determined from the relic annihilations of either the neutralino or stau (if below the dotted line) and $\Omega_{3 / 2} h^{2}=\left(m_{3 / 2} / m_{\chi, \tilde{\tau}_{1}}\right) \Omega_{\chi, \tilde{\tau}_{1}} h^{2}$. For $A_{0}=2 m_{0}$, there is a 'normal' WMAP co-annihilation strip where the LSP is a neutralino. Below the strip there is a wedge where the LSP is a stau (shaded) and below that, the LSP is the gravitino, with a density below that in Eq. (2.1).

An interesting extension of minimal supergravity is one where terms proportional $W^{(2)}$ are added to the Kähler potential as in the Giudice-Masiero mechanism [20]. For example, consider an additional contribution to $K$,

$$
\Delta K=c_{H} H_{1} H_{2}+\text { h.c. }
$$



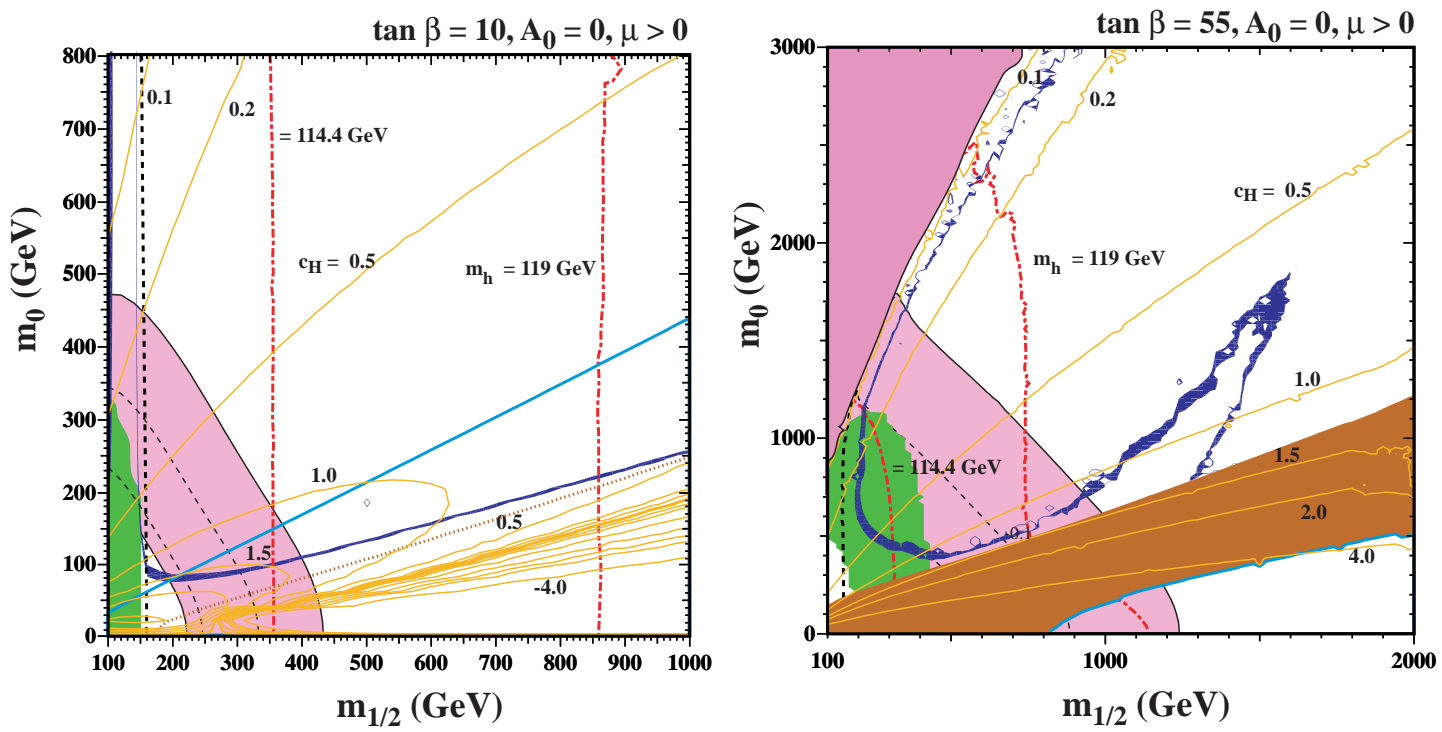

Figure 3: The $\left(m_{1 / 2}, m_{0}\right)$ planes for CMSSM based on a GM supergravity model with $A_{0}=0, c_{H} \neq 0$ and with $\tan \beta=10$ (left) and $\tan \beta=55$ (right). The meaning of the curves and shaded regions are the same as in Fig. 1. However, here we show the contours of the required value of $c_{H}$ in order to maintain the fixed value of $\tan \beta$ across the plane.

where $c_{H}$ is a constant, and $H_{1,2}$ are the usual MSSM Higgs doublets. The effect of $\Delta K$ is manifest on the boundary conditions for both $\mu$ and the $B$ term at the supersymmetry breaking input scale, $M_{\text {in }}$. The $\mu$ term is shifted to

$$
\mu+c_{H} m_{0}
$$

but, for our purposes this shift is irrelevant since the input value of $\mu$ is arbitrary and the low energy value is fixed by the minimization condition (1.3). The boundary condition on $\mu B$ shifts from $\mu B_{0}$ to

$$
\mu B_{0}+2 c_{H} m_{0}^{2}
$$

Therefore, it is possible to solve for $B$ at the weak scale for an arbitrary $\tan \beta$, and still satisfy the GUT scale supergravity boundary condition, thus solving for $c_{H}$ [19]. Therefore, relaxing the condition between $A_{0}$ and $B_{0}$ and considering $\tan \beta$ as an input, as is done in the CMSSM, is equivalent to "switching on" the coefficient $c_{H}$ in Eq. (2.2). In other words, for a given value of $\tan \beta$ and $A_{0}$, at each point $\left(m_{1 / 2}, m_{0}\right)$ there may exist a single value of $c_{H}$ respecting the mSUGRA boundary condition for $B_{0}$

$$
B_{0}=A_{0}-m_{0}+2 c_{H} m_{0}^{2} / \mu_{0}
$$

We display the iso- $c_{H}$ contours in Fig. 3 for $A_{0}=0$ and $\tan \beta=10$ and 55 [19]. As one can see, over much of the plane, the values of $c_{H}$ are reasonably small. Also note that unlike the CMSSM, below the light blue diagonal line, the gravitino is the LSP. 

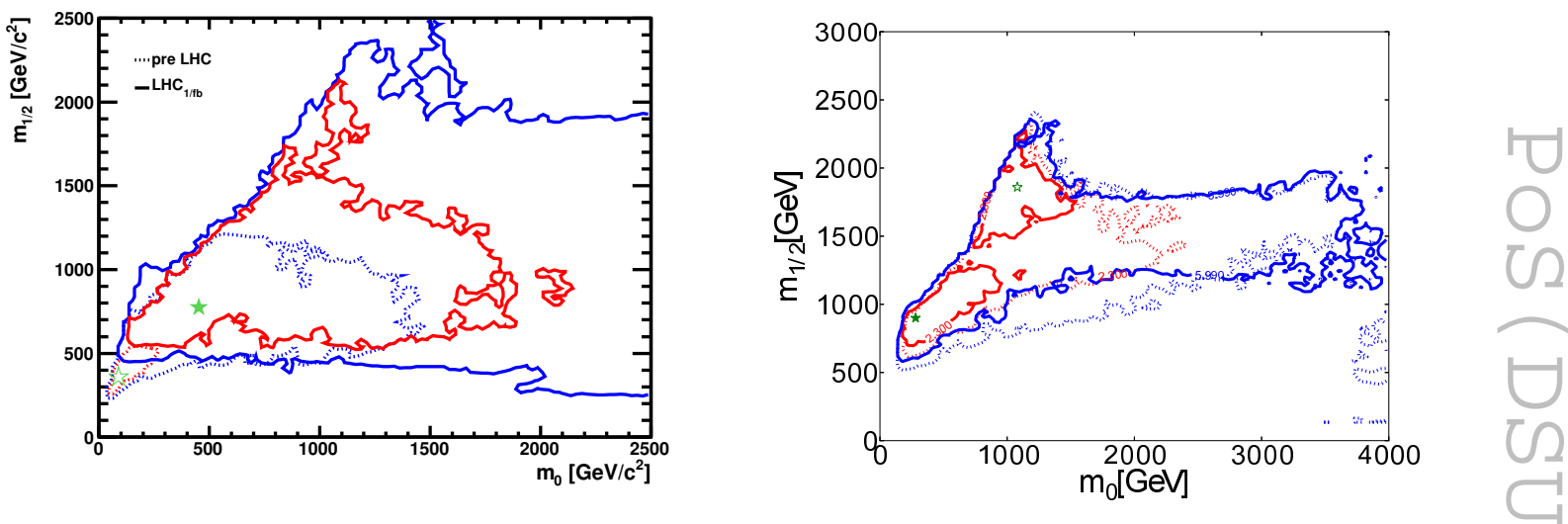

Figure 4: The $\left(m_{0}, m_{1 / 2}\right)$ planes in the CMSSM. Left: the best-fit point [28] after incorporation of the $L H C_{1 / \mathrm{fb}}$ constraints is indicated by a filled green star, and the pre-LHC fit [27] by an open star. The 68 and 95\% CL regions are indicated in red and blue, respectively, the solid lines including the $L H C_{1 / \mathrm{fb}}$ data and the dotted lines showing the pre-LHC fits [28]. Right: The results of the $5 \mathrm{fb}^{-1}$ fits [21] are indicated by solid lines and filled stars, and previous fits based on $\sim 1 /$ fb of LHC data are indicated by dashed lines and open stars.

\section{Effect of the Results from the LHC}

By the end of 2011, LHC searches for jets and missing $E_{T}$ with $5 \mathrm{fb}^{-1}$ of data [16], made strong inroads on the parameter space of the CMSSM [21, 22]. This limit is shown by the solid purple curve (labelled LHC) in Figs. 1 and 2 and excludes points to the left of this curve. There have also been analyses of the CMSSM on the implications of a Higgs boson weighing $\sim 125$ $\mathrm{GeV}[23,24,25,22]$. In [21], the LHC results were incorporated in a Markov-Chain Monte Carlo analyses in the Mastercode (MC) framework [26] which is used to sample the SUSY parameter space, and construct the $\chi^{2}$ probability function, $P\left(\chi^{2}, N_{\text {dof }}\right)$. This provides a quantitative measure for the quality-of-fit such that $P\left(\chi^{2}, N_{\text {dof }}\right)$ can be used to estimate the absolute probability with which the CMSSM describes the experimental data.

Some results from the MC analyses are shown in Fig. 4. In the left panel, a comparison of the pre-LHC predictions of in the $m_{0}, m_{1 / 2}$ parameter plane are shown in comparison with the LHC results at $1 / \mathrm{fb}[27,28]$. The best-fit point at $1 / \mathrm{fb}$ is shown as a filled star and 68 and $95 \% \mathrm{CL}$ regions are shown as red and blue lines, respectively and correspond to $\Delta \chi^{2}=2.3$ (red) and 5.99 (blue) relative to the best fit point. The pre-LHC results, taken from [27], are displayed as an open star and dotted lines. The right panel compares the $1 / \mathrm{fb}$ results with the additional constraint of a Higgs with a mass of $125 \mathrm{GeV}$, and the 5/fb [21] results. The main effect of the LHC constraint is to shave off a slice of points at low $m_{1 / 2}$ and moderate $m_{0}$. 

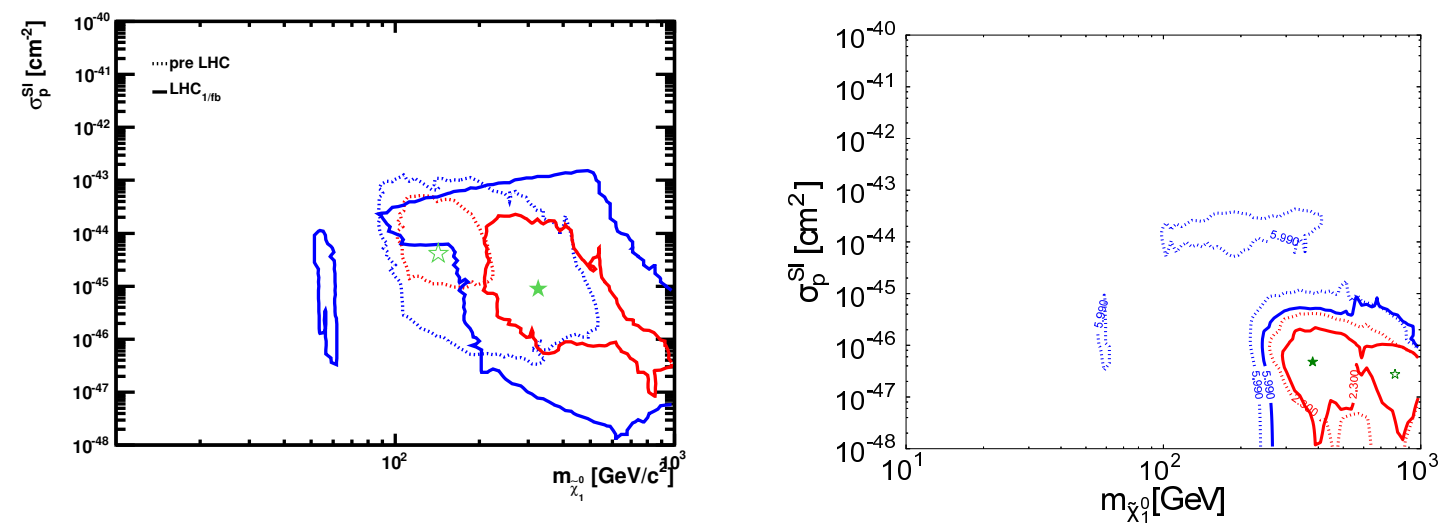

Figure 5: The $\left(m_{\chi_{1}}, \sigma_{p}^{s s i}\right)$ planes in the CMSSM showing the $68 \%$ and $95 \% C L$ contours (red and blue, respectively) in the CMSSM. Left: the solid lines are for fits including the XENON100 [30] and $\mathrm{LHC}_{1 / \mathrm{fb}}$ data, whereas the dotted lines include only the pre-LHC data [28]. Right: The results of the $5 \mathrm{fb}^{-1}$ fits are indicated by solid lines and filled stars, and previous fits based on $\sim 1 / f b$ of LHC data are indicated by dashed lines and open stars.

We see in Fig. 4 that the 95\% CL region in the CMSSM extends to $m_{0} \sim 4000 \mathrm{GeV}$ and $m_{1 / 2} \sim 2400 \mathrm{GeV}$. Note that the CMSSM fit features two disconnected 68\% CL 'islands', the one at lower $m_{0}$ and $m_{1 / 2}$ corresponding to the stau coannihilation region, and that at larger $m_{0}$ and $m_{1 / 2}$ corresponding to the rapid-annihilation funnel region. However, the MC sampling also includes a few intermediate points that also have $\Delta \chi^{2}<2.30$, reflecting the relative flatness of the global $\chi^{2}$ function along the band between $\left(m_{0}, m_{1 / 2}\right) \sim(300,900) \mathrm{GeV}$ and $\sim(1100,1900) \mathrm{GeV}$. Typically, these points sit in rapid-annihilation funnels, and it is possible that a more complete sampling might reveal a lower 'isthmus' connecting these islands.

Fig. 5 displays the best-fit points and the 68\% and 95\% CL contours (red and blue, respectively) in the $\left(m_{\chi}, \sigma_{p}^{s s i}\right)$ plane in the CMSSM [27, 28, 21]. As in Fig. 4, the pre-LHC results are shown compared with the $1 / \mathrm{fb}$ results on the left. In the right panel, the 5/fb results are compared to the $1 / \mathrm{fb}$ results including a Higgs mass at $125 \mathrm{GeV}$. The solid lines on the right correspond to the global fit to the LHC results and include new results on $B_{s} \rightarrow \mu^{+} \mu^{-}$[29] and new XENON100 data set [30]. We see that the region of the $\left(m_{\chi}, \sigma_{p}^{s s i}\right)$ favored in the CMSSM is now more restricted, in particular at small $m_{\chi}$. The main effect of the new XENON100 constraint has been to remove focus points with large $\sigma_{p}^{s s i}$ that were previously allowed at the $95 \% \mathrm{CL}$.

\section{The impact of a $125 \mathrm{GeV}$ Higgs mass on the CMSSM}

In Ref. [31], the CMSSM parameter space was scanned over the ranges $100 \mathrm{GeV}<m_{1 / 2}<$ 

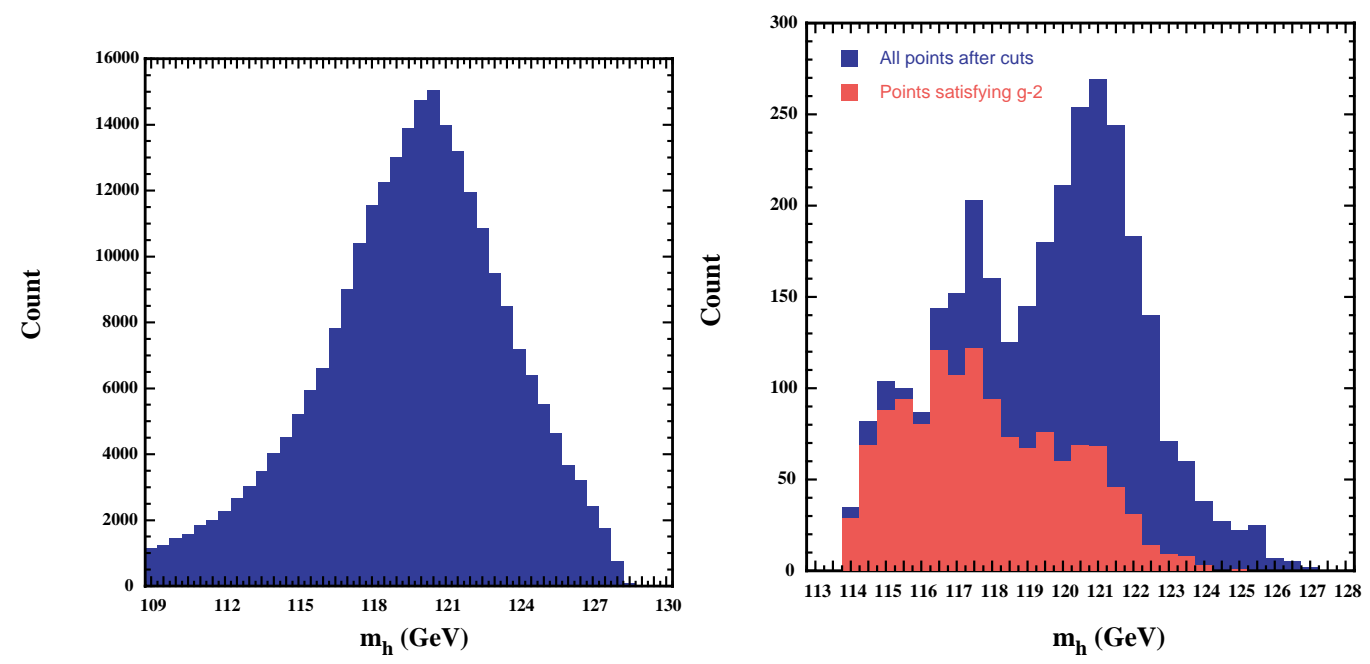

Figure 6: The distribution of the mass of the lightest neutral Higgs boson, $m_{h}$, in the CMSSM (a) before applying the accelerator cuts and the WMAP relic density constraint, and (b) after applying these constraints. In the latter case, the red (light) histogram shows the points favored by the optional $g_{\mu}-2$ constraint.

$2 \mathrm{TeV}, m_{0}<2 \mathrm{TeV},\left|A_{0} / m_{1 / 2}\right|<3,2<\tan \beta<58$ and $\mu>0$ resulting in the histogram shown Fig. 6 for $m_{h}$. On the left, the only cut made in the sample is the requirement that the LSP is a neutralino and 260,000 points are included. On the right, accelerator and relic density constraints are applied leaving 3075 points in the sample. The still smaller sample shown in red requires that the $g_{\mu}-2$ discrepancy is resolved. Because of the logarithmic dependence of $m_{h}$ on $m_{1 / 2}$, even a modest change in the position of the peak would require increasing the upper limit on $m_{1 / 2}$ substantially. While it is encouraging that the range found in [31] includes the value $m_{h} \sim 125 \mathrm{GeV}$, it is equally clear that this value is far from the mode of the histogram. Therefore, to accommodate a Higgs mass of $125 \mathrm{GeV}$, higher supersymmetry breaking masses will required.

From the results seen in Fig. 1, it is clear that higher masses alone will not be sufficient for increasing the Higgs mass to $125 \mathrm{GeV}$. A further increase in $m_{h}$ is possible when $A_{0}>0$. In the left panel of Fig. 7, the $m_{1 / 2}, m_{0}$ plane is shown for $\tan \beta=40$ and $A_{0}=2.5 m_{0}$. In addition to the region in the lower right due to a stau LSP, there is a similarly shaded region in the upper left due to a stop LSP. Along the boundary of this region, there is a WMAP strip caused by neutralino-stop co-annihilations [32]. However it sits in a region excluded by $b \rightarrow s \gamma$ (shaded green). For these values of $\tan \beta$ and $A_{0}$, the stau coannihilation region extends to larger Higgs masses, including $125 \mathrm{GeV}$.

The consequences for direct detection are rather dramatic. As we saw in Fig. 5, the predicted elastic scattering cross section is significantly below the current XENON100 limits [30]. This is seen again in the right panel of Fig. 7, which shows the spin independent elastic cross section along the WMAP coannihilation strip for $\tan \beta=40$ and $A_{0}=0$ and $2.5 m_{0}$.

\section{Summary}

The discovery of the Higgs Boson has had an immediate impact on our understanding of 

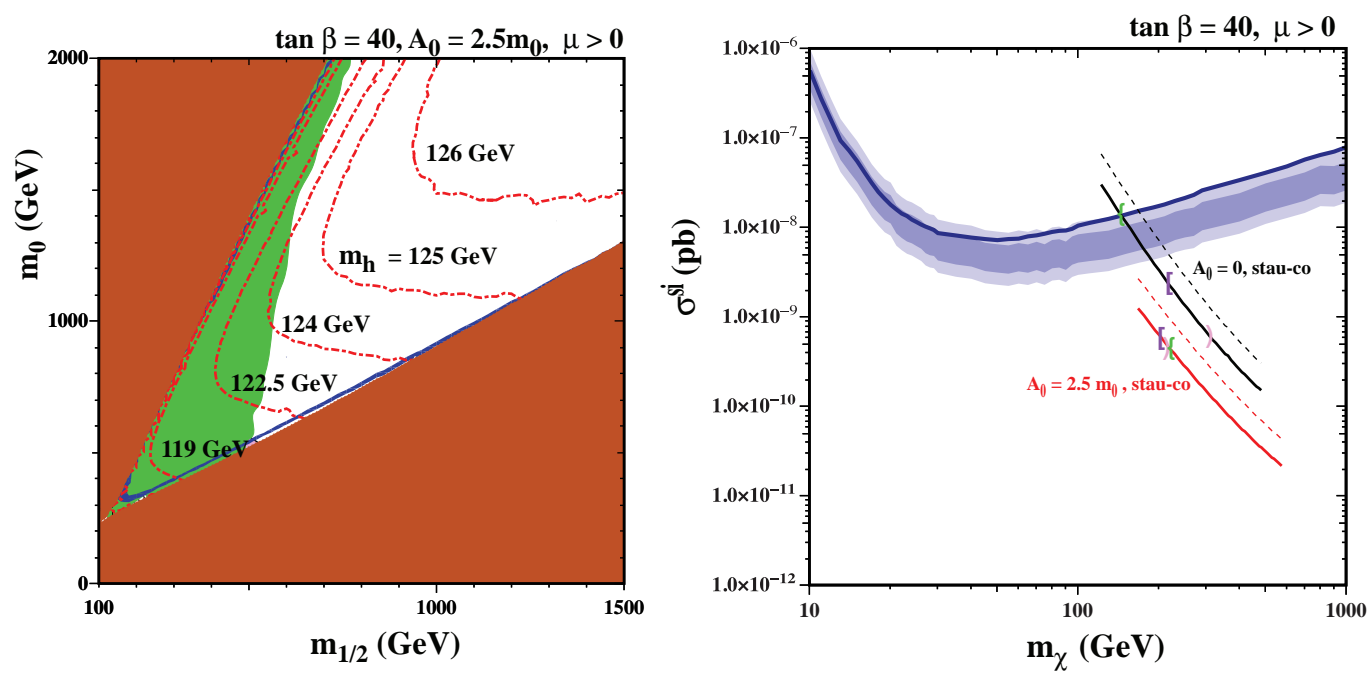

Figure 7: Left: As in Fig. 1 for $\tan \beta=40$ and $A_{0}=2.5 m_{0}$. Right: The spin independent elastic cross section as a function of the neutralino mass along the stau coannihilation strip as compared to the XENON100 exclusion [30]. The portions of these strips allowed by the LHC missing-energy searches [16] are indicated by (purple) square brackets: [ and the portions favored by $g_{\mu}-2$ are indicated by (pink) parentheses: ). The lower bounds on $m_{1 / 2}$ along these strips due to $b \rightarrow s \gamma$ are indicated by green brackets $\{$.

supersymmetric dark matter. Despite the fact that a $125 \mathrm{GeV}$ mass for the Higgs boson fits the predictions of simple supersymmetric models, there is no question that a Higgs mass of $125 \mathrm{GeV}$ presents a challenge for CMSSM-like models. Not only are supersymmetric particle mass scales pushed up (consistent with the lack of discovery of supersymmetry at the LHC) but large values of the trilinear coupling are preferred. The probability that the $g_{\mu}-2$ discrepancy is resolved by supersymmetry is also greatly diminished as is the improvement by supersymmetry to the global fit of electroweak observables over the Standard Model [21]. Finally, predictions for the elastic scattering cross sections for the direct detection of dark matter are pushed well below current experimental limits.

\section{References}

[1] H. Goldberg, Phys. Rev. Lett. 50 (1983) 1419; J. Ellis, J. Hagelin, D. Nanopoulos, K. Olive and M. Srednicki, Nucl. Phys. B 238 (1984) 453.

[2] E. Cremmer, B. Julia, J. Scherk, P. van Nieuwenhuizen, S. Ferrara and L. Girardello, Phys. Lett. B 79, 231 (1978); E. Cremmer, B. Julia, J. Scherk, S. Ferrara, L. Girardello and P. van Nieuwenhuizen, Nucl. Phys. B 147, 105 (1979); For reviews, see: H. P. Nilles, Phys. Rep. 110 (1984) 1; A. Brignole, L. E. Ibanez and C. Munoz, arXiv:hep-ph/9707209, published in Perspectives on supersymmetry, ed. G. L. Kane, pp. 125-148.

[3] A. H. Chamseddine, R. L. Arnowitt and P. Nath, Phys. Rev. Lett. 49, 970 (1982); R. L. Arnowitt, A. H. Chamseddine and P. Nath, Phys. Rev. Lett. 50, 232 (1983); R. Arnowitt, A. H. Chamseddine and P. Nath, arXiv:1206.3175 [physics.hist-ph].

[4] R. Barbieri, S. Ferrara and C. A. Savoy, Phys. Lett. B 119, 343 (1982). 
[5] V. D. Barger, M. S. Berger and P. Ohmann, Phys. Rev. D 49 (1994) 4908 [arXiv:hep-ph/9311269]; W. de Boer, R. Ehret and D. I. Kazakov, Z. Phys. C 67 (1995) 647 [arXiv:hep-ph/9405342]; M. Carena, J. R. Ellis, A. Pilaftsis and C. E. Wagner, Nucl. Phys. B 625 (2002) 345 [arXiv:hep-ph/0111245].

[6] M. Drees and M. M. Nojiri, Phys. Rev. D 47 (1993) 376 [arXiv:hep-ph/9207234]; H. Baer and M. Brhlik, Phys. Rev. D 53 (1996) 597 [arXiv:hep-ph/9508321]; Phys. Rev. D 57 (1998) 567 [arXiv:hep-ph/9706509]; H. Baer, M. Brhlik, M. A. Diaz, J. Ferrandis, P. Mercadante, P. Quintana and X. Tata, Phys. Rev. D 63 (2001) 015007 [arXiv:hep-ph/0005027].

[7] G. L. Kane, C. F. Kolda, L. Roszkowski and J. D. Wells, Phys. Rev. D 49 (1994) 6173 [arXiv:hep-ph/9312272]; J. R. Ellis, T. Falk, K. A. Olive and M. Schmitt, Phys. Lett. B 388 (1996) 97 [arXiv:hep-ph/9607292]; Phys. Lett. B 413 (1997) 355 [arXiv:hep-ph/9705444]; J. R. Ellis, T. Falk, G. Ganis, K. A. Olive and M. Schmitt, Phys. Rev. D 58 (1998) 095002 [arXiv:hep-ph/9801445]; V. D. Barger and C. Kao, Phys. Rev. D 57 (1998) 3131 [arXiv:hep-ph/9704403]; J. R. Ellis, T. Falk, G. Ganis and K. A. Olive, Phys. Rev. D 62 (2000) 075010 [arXiv:hep-ph/0004169].

[8] J. R. Ellis, T. Falk, G. Ganis, K. A. Olive and M. Srednicki, Phys. Lett. B 510 (2001) 236 [arXiv:hep-ph/0102098].

[9] V. D. Barger and C. Kao, Phys. Lett. B 518 (2001) 117 [arXiv:hep-ph/0106189]; L. Roszkowski, R. Ruiz de Austri and T. Nihei, JHEP 0108 (2001) 024 [arXiv:hep-ph/0106334]; A. Djouadi, M. Drees and J. L. Kneur, JHEP 0108 (2001) 055 [arXiv:hep-ph/0107316]; U. Chattopadhyay, A. Corsetti and P. Nath, Phys. Rev. D 66 (2002) 035003 [arXiv:hep-ph/0201001]; J. R. Ellis, K. A. Olive and Y. Santoso, New Jour. Phys. 4 (2002) 32 [arXiv:hep-ph/0202110]; H. Baer, C. Balazs, A. Belyaev, J. K. Mizukoshi, X. Tata and Y. Wang, JHEP 0207 (2002) 050 [arXiv:hep-ph/0205325]; R. Arnowitt and B. Dutta, arXiv:hep-ph/0211417.

[10] J. R. Ellis, K. A. Olive, Y. Santoso and V. C. Spanos, Phys. Lett. B 565 (2003) 176 [arXiv:hep-ph/0303043]; H. Baer and C. Balazs, JCAP 0305, 006 (2003) [arXiv:hep-ph/0303114]; A. B. Lahanas and D. V. Nanopoulos, Phys. Lett. B 568, 55 (2003) [arXiv:hep-ph/0303130]; U. Chattopadhyay, A. Corsetti and P. Nath, Phys. Rev. D 68, 035005 (2003) [arXiv:hep-ph/0303201]; C. Munoz, Int. J. Mod. Phys. A 19, 3093 (2004) [arXiv:hep-ph/0309346]; R. Arnowitt, B. Dutta and B. Hu, arXiv:hep-ph/0310103. J. Ellis and K. A. Olive, In *Bertone, G. (ed.): Particle dark matter* 142-163 [arXiv:1001.3651 [astro-ph.CO]].

[11] E. Komatsu et al. [WMAP Collaboration], Astrophys. J. Suppl. 192 (2011) 18 [arXiv:1001.4538 [astro-ph.CO]].

[12] J. Ellis, T. Falk, and K.A. Olive, Phys. Lett. B444 (1998) 367 [arXiv:hep-ph/9810360]; J. Ellis, T. Falk, K.A. Olive, and M. Srednicki, Astr. Part. Phys. 13 (2000) 181 [Erratum-ibid. 15 (2001) 413] [arXiv:hep-ph/9905481].

[13] Joint LEP 2 Supersymmetry Working Group, Combined LEP Chargino Results, up to $208 \mathrm{GeV}$, http://lepsusy.web.cern.ch/lepsusy/www/inos_ moriond01/charginos_pub.html.

[14] S. Chen et al. [CLEO Collaboration], Phys. Rev. Lett. 87 (2001) 251807 [arXiv:hep-ex/0108032]; P. Koppenburg et al. [Belle Collaboration], Phys. Rev. Lett. 93 (2004) 061803 [arXiv:hep-ex/0403004]. B. Aubert et al. [BaBar Collaboration], arXiv:hep-ex/0207076; E. Barberio et al. [Heavy Flavor Averaging Group (HFAG)], arXiv:hep-ex/0603003.

[15] [The Muon g-2 Collaboration], Phys. Rev. Lett. 92 (2004) 161802, hep-ex/0401008; G. Bennett et al. [The Muon g-2 Collaboration], Phys. Rev. D 73 (2006) 072003 [arXiv:hep-ex/0602035]. 
[16] ATLAS Collaboration, https://cdsweb.cern.ch/record/1432199/files/ATLAS-CONF-2012-033.pdf; S. Chatrchyan et al. [CMS Collaboration], JHEP 1210, 018 (2012) [arXiv:1207.1798 [hep-ex]]; arXiv:1207.1898 [hep-ex].

[17] J. L. Feng, K. T. Matchev and T. Moroi, Phys. Rev. D 61 (2000) 075005 [arXiv:hep-ph/9909334].

[18] J. R. Ellis, K. A. Olive, Y. Santoso and V. C. Spanos, Phys. Lett. B 573 (2003) 162 [arXiv:hep-ph/0305212], and Phys. Rev. D 70 (2004) 055005 [arXiv:hep-ph/0405110].

[19] E. Dudas, Y. Mambrini, A. Mustafayev and K. A. Olive, Eur. Phys. J. C 72, 2138 (2012) [arXiv:1205.5988 [hep-ph]].

[20] G. F. Giudice and A. Masiero, Phys. Lett. B 206, 480 (1988).

[21] O. Buchmueller, R. Cavanaugh, M. Citron, A. De Roeck, M. J. Dolan, J. R. Ellis, H. Flacher and S. Heinemeyer et al., arXiv:1207.7315 [hep-ph].

[22] C. Strege, G. Bertone, D. G. Cerdeno, M. Fornasa, R. R. de Austri and R. Trotta, JCAP 1203 (2012) 030 [arXiv:1112.4192 [hep-ph]]; P. Bechtle, T. Bringmann, K. Desch, H. Dreiner, M. Hamer, C. Hensel, M. Kramer and N. Nguyen et al., JHEP 1206, 098 (2012) [arXiv:1204.4199 [hep-ph]]; A. Fowlie, M. Kazana, K. Kowalska, S. Munir, L. Roszkowski, E. M. Sessolo, S. Trojanowski and Y. -L. S. Tsai, arXiv:1206.0264 [hep-ph]; T. Li, J. A. Maxin, D. V. Nanopoulos and J. W. Walker, arXiv:1206.2633 [hep-ph] and references therein.

[23] O. Buchmueller, et al., Eur. Phys. J. C 72 (2012) 2020 [arXiv:1112.3564 [hep-ph]].

[24] H. Baer, V. Barger and A. Mustafayev, Phys. Rev. D 85, 075010 (2012) [arXiv:1112.3017 [hep-ph]]; J. L. Feng, K. T. Matchev and D. Sanford, Phys. Rev. D 85, 075007 (2012) [arXiv:1112.3021 [hep-ph]]; T. Li, J. A. Maxin, D. V. Nanopoulos and J. W. Walker, Phys. Lett. B 710 (2012) 207 [arXiv:1112.3024 [hep-ph]]; S. Heinemeyer, O. Stal and G. Weiglein, Phys. Lett. B 710, 201 (2012) [arXiv:1112.3026 [hep-ph]]; A. Arbey, M. Battaglia, A. Djouadi, F. Mahmoudi and J. Quevillon, Phys. Lett. B 708 (2012) 162 [arXiv:1112.3028 [hep-ph]]; P. Draper, P. Meade, M. Reece and D. Shih, Phys. Rev. D 85, 095007 (2012) [arXiv:1112.3068 [hep-ph]]; S. Akula, B. Altunkaynak, D. Feldman, P. Nath and G. Peim, Phys. Rev. D 85 (2012) 075001 [arXiv:1112.3645 [hep-ph]]; M. Kadastik, K. Kannike, A. Racioppi and M. Raidal, JHEP 1205 (2012) 061 [arXiv:1112.3647 [hep-ph]]; J. Cao, Z. Heng, D. Li and J. M. Yang, Phys. Lett. B 710 (2012) 665 [arXiv:1112.4391 [hep-ph]]; L. Aparicio, D. G. Cerdeno and L. E. Ibanez, JHEP 1204, 126 (2012) [arXiv:1202.0822 [hep-ph]]; H. Baer, V. Barger and A. Mustafayev, JHEP 1205 (2012) 091 [arXiv:1202.4038 [hep-ph]]; C. Balazs, A. Buckley, D. Carter, B. Farmer and M. White, arXiv:1205.1568 [hep-ph]; D. Ghosh, M. Guchait, S. Raychaudhuri and D. Sengupta, Phys. Rev. D 86, 055007 (2012) [arXiv:1205.2283 [hep-ph]].

[25] J. Ellis and K. A. Olive, Eur. Phys. J. C 72, 2005 (2012) [arXiv:1202.3262 [hep-ph]].

[26] For more information and updates, please see http://cern. ch/mastercode/.

[27] O. Buchmueller et al., Eur. Phys. J. C 71 (2011) 1583 [arXiv:1011.6118 [hep-ph]]; O. Buchmueller et al., Eur. Phys. J. C 71 (2011) 1634 [arXiv:1102.4585 [hep-ph]].

[28] O. Buchmueller, R. Cavanaugh, A. De Roeck, M. J. Dolan, J. R. Ellis, H. Flacher, S. Heinemeyer and G. Isidori et al., Eur. Phys. J. C 72, 1878 (2012) [arXiv:1110.3568 [hep-ph]].

[29] G. Aad et al. [ATLAS Collaboration], Phys. Lett. B 713, 387 (2012) [arXiv:1204.0735 [hep-ex]]; T. Aaltonen et al. [CDF Collaboration], Phys. Rev. Lett. 107, 239903 (2011) [Phys. Rev. Lett. 107, 191801 (2011)] [arXiv:1107.2304 [hep-ex]]; updated results presented at Aspen in Feb. 2012 by M. Rescigno, https://indico.cern.ch/getFile.py/ 
access?contribId $=28$ \&session $I d=7 \&$ res $I d$ $=1 \&$ material Id=slides\&conf Id=143360.; S. Chatrchyan et al. [CMS Collaboration], Phys. Rev. Lett. 107, 191802 (2011) [arXiv:1107.5834 [hep-ex]]; R. Aaij et al. [LHCb Collaboration], Phys. Lett. B 699 (2011) 330 [arXiv:1103.2465 [hep-ex]]; arXiv:1203.4493 [hep-ex]; For an official combination of the ATLAS, CMS and LHCb results, see: ATLAS, CMS, and LHCb Collaborations, http://cdsweb.cern.ch/record/1452186/files/LHCb-CONF-2012-017.pdf.

[30] E. Aprile et al. [XENON100 Collaboration], Phys. Rev. Lett. 107 (2011) 131302 [arXiv:1104.2549 [astro-ph.CO]].

[31] J. R. Ellis, D. V. Nanopoulos, K. A. Olive and Y. Santoso, Phys. Lett. B 633, 583 (2006) [arXiv:hep-ph/0509331].

[32] C. Boehm, A. Djouadi and M. Drees, Phys. Rev. D 62 (2000) 035012 [arXiv:hep-ph/9911496]; J. R. Ellis, K. A. Olive and Y. Santoso, Astropart. Phys. 18 (2003) 395 [arXiv:hep-ph/0112113]; K. Huitu, L. Leinonen and J. Laamanen, Phys. Rev. D 84, 075021 (2011) [arXiv:1107.2128 [hep-ph]]. 